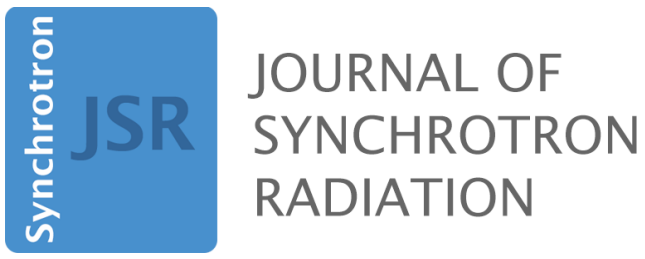

Volume 22 (2015)

Supporting information for article:

Compound focusing mirror and X-ray waveguide optics for coherent imaging and nano-diffraction

Tim Salditt, Markus Osterhoff, Martin Krenkel, Robin N. Wilke, Marius Priebe, Matthias Bartels, Sebastian Kalbfleisch and Michael Sprung 


\title{
Compound focusing mirror and x-ray waveguide optics for coherent imaging and nano-diffraction: Supplemental Information
}

\author{
Tim Salditt, ${ }^{a *}$ Markus Osterhoff,${ }^{a}$ Martin Krenkel,${ }^{a}$ Robin N. Wilke ${ }^{a}$ \\ Marius Priebe, ${ }^{a}$ Matthias Bartels,${ }^{a}$ Sebastian Kalbfleisch $^{a}$ And \\ Michael Sprung ${ }^{b}$ \\ ${ }^{a}$ Institut für Röntgenphysik, Georg-August-Universität Göttingen, 37077 Göttingen \\ (Germany), and ${ }^{b}$ Desy, Notkestrasse 85, 22607 Hamburg (Germany). \\ E-mail: tsaldit@gwdg.de
}

\begin{abstract}
In this supplemental material, we give more information on the instrumentation of the coherent nano-focus endstation GINIX (Göttingen Instrument for Nano-Imaging with X-rays), installed at the P10 coherence beamline of the PETRA III synchrotron at DESY, Hamburg. The coherent nano-focus imaging approach described in the main article has been implemented based on the Kirkpatrick-Baez (KB) mirror and x-ray waveguide optical system of this endstation, designed and dedicated for this purpose. The results for the different imaging modalities and applications have been obtained with the instrument components and parameters, as further detailed here for matters of completeness, and as practical information for users of the instrument.
\end{abstract}

PREPRINT: Journal of Synchrotron Radiation

A Journal of the International Union of Crystallography 


\section{Instrumentation}
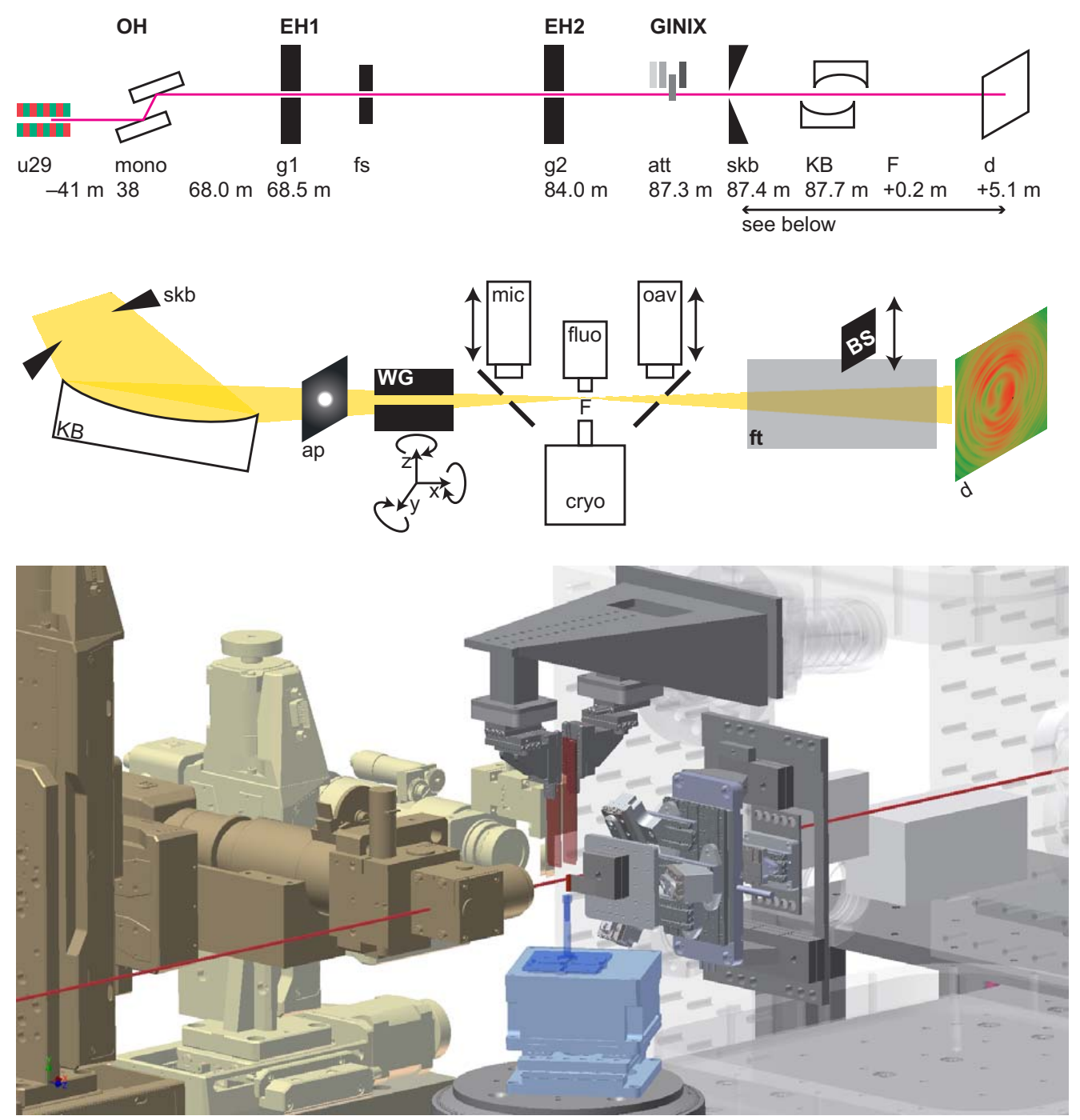

Fig. 1. Instrumentation and setup of the GINIX endstation. (top) Schematic of the beamline, with undulator (u29), double crystal monochromator (mono), entrance slits $(\mathrm{g} 1, \mathrm{~g} 2)$ to the experimental hutches, automated attenuators (att), KB clean-up slits (skb), the KB mirrors, the focal plane (F) and the detection plane (d), with the repective distances. (middle) Close-up of the compound optical system with $\mathrm{KB}$, beam selection aperture (ap), waveguide (WG), on-axis optical microscopes (mic,oav), an optional fluorescence detector (fluo), the cryojet sample environment (cryo), and the flight tube (ft) containing two beamstop holders (BS). (bottom) CAD drawing of the sample environment with KB box (light gray), waveguide and optics holders (dark gray), sample stage (blue), and optical microscopes, providing the on-axis views in downstream (from KB towards waveguide) orientation (yellow), and in upstream (from detector towards the sample) orientation (brown), IUGresqseetiveredy.2.1.6: 2014/10/01 
The GINIX endstation is installed in the second experimental hutch (EH2) of the PETRAIII/P10 beamline, at a distance of about $87 \mathrm{~m}$ behind the the $5 \mathrm{~m}$ undulator (period $\lambda=29 \mathrm{~mm}$, max. field $B=0.81 \mathrm{~T}$ ), providing a source (low- $\beta$ setting) of $36 \mu \mathrm{m} \times 6 \mu \mathrm{m}(\mathrm{h} \times \mathrm{v}, 1 \sigma)$ with a divergence of $28 \mu \mathrm{rad} \times 3.7 \mu \mathrm{rad}(\mathrm{h} \times \mathrm{v}, 1 \sigma)$, and maximum brilliance of $B \geq 510^{20} \mathrm{ph} / \mathrm{s} / \mathrm{mrad}^{2} / \mathrm{mm} / 0.1 \% \mathrm{bw}$ in the first harmonic at $\simeq 6 \mathrm{keV}$ (Leupold \& Sprung, 2008; Balewski et al., 2004). Following the primary $(14 \mathrm{~m})$ and secondary slit system $(28 \mathrm{~m})$, the vertically deflecting and liquid nitrogen cooled monochromator $(38 \mathrm{~m})$ is the first optical elements to receive the white beam. The following options are available: high heat-load, fixed-exit double crystal monochromators $(\operatorname{Si}(111) / \mathrm{Si}(311))$ or channel-cut $(\mathrm{Si}(111))$, installed more recently to avoid the pitch vibrations observed for the first system. The channel cut is thus the best option for vibrational stability, the double crystal yields slightly higher flux and facilitates fast changes of photon energy, e.g. for resonant diffraction and imaging applications, see below. A pair of horizontally deflecting mirrors $(\simeq 41 \mathrm{~m}, R h$ or $P t)$ can be used for higher harmonic suppression. For the KB-system of GINIX, it was found, however, that the harmonics suppression by the two KB mirrors was sufficient, so that the horizontal mirrors can be omitted to better preserve the wavefront. A beam position monitor (FMB Oxford) is installed at the entrance of EH1. The beam in both hutches can be set with entrance slits $G 1$ and $G 2$ (custom made slits system by Desy, motor controllers by Galil Inc.), at $68 \mathrm{~m}$ and $84 \mathrm{~m}$, respectively, followed by monitor counters (mon1/mon2).

Endstation installations: The endstation containing the KB vacuum vessel, the sample environment and a first detector bench is mounted on a motorized optical table (DES-104, IDT) in EH2, with a mass of $1300 \mathrm{~kg}$ and a breadboard size of $1.5 \times 1.0 \mathrm{~m}$, which can be moved on air pads from a parking position to the beam position, made ready to use (cabling), and aligned using first an optical autocollimator (Möller-Wedel 
AKR 300/65/14,7) and then the x-ray beam, within less than a day (Kalbfleisch, 2012). The instrument's entrance slit system equipped with hybrid metal-single-crystal blades (Xenocs, France), in front of the KB mirrors serves as additional reference for the lateral position. The beam path to the motorized rear detector bench at $\geq 5 \mathrm{~m}$ behind the $\mathrm{KB}$ focal plane is evacuated by a flight tube with exit diameter of $180 \mathrm{~mm}$ (Kapton foil), two integrated beamstop holders, different nose pieces selected in view of the required sample positions (defocus) and eventual need for front detectors, as well as two types of entrance window, i.e 100mm diameter Kapton windows for diffraction, and squared $S i_{3} N_{4}$ foils of $7 \mathrm{~mm}$ side length for holographic imaging. An additional beamstop holder can be used to capture the primary beam before entering the flight tube, which is of advantage for nano-diffraction experiments, and a necessity when using the pixel detectors on the front detector bench.

Detectors: For scanning nano-diffraction (SAXS) experiments, the multi-module hybrid pixel detectors with single photon counting capability (Rossi et al., 2006) are routinely available at P10 (rear detector bench), namely the Pilatus 300k (Dectris) and a Maxipix $(\mathrm{ESRF})$ with $4 \times 4$ modules. Further pixel detector systems which have been tested and fully implemented in the GINIX instrument control are the novel Lambda detector (Desy) (Pennicard et al., 2012; Wilke et al., 2014) and a Mixed-Mode Pixel Array Detector (MM-PAD) (Giewekemeyer et al., 2014). For scanning nano-diffraction in the WAXS range (front detector bench), the Pilatus 1M /4M (Dectris) detectors can be reserved from the detector pool for installation directly on the GINIX table. For holographic imaging, smaller pixel size $p$ and more pixels are needed to cover the full field, and inter-module gaps should be avoided. The first detector for that purpose at GINIX was a direct-illuminated CCDs (Roper Scientific LCX), now replaced by a similar system (Pixis-XO) with $2048 \times 2048$ pixels and $13.4 \mu \mathrm{m}$ pixel size, but limitations in dynamic range and (full well capacity) and read-out speed make indirect

IUCr macros version 2.1.6: 2014/10/01 
detection based scintillators in combination with high quality optical cameras more attractive. The instrument's work horse is a fibre coupled sCMOS detector (Photonic Science) with a pixel size of $P=6.54 \mu \mathrm{m}$, and for higher resolution, a custom-built scintillator microscope based detection system (Optique Peter) equipped with a $20 \mu \mathrm{m}$ LuAG:Ce scintillator (Crytur) with a half-period resolution of $1.05 \mu \mathrm{m}$ imaged by $10-$ or 4-fold magnifying objectives (Olympus) to a PCO2000 CCD (2048 by 2048 pixels, $7.4 \mu \mathrm{m}$ pixel size), resulting in effective pixel sizes of $0.74 \mu \mathrm{m}$ or $1.85 \mu \mathrm{m}$, for the two objectives, respectively (Olendrowitz et al., 2012; Bartels et al., 2012). The exposure time of the detectors is controlled by piezo-based shutter (CEDRAT FPS400M) with a response time of $1.01 \mathrm{~ms}$, which in addition serves the purpose to protect the sample from radiation dose during motor movement or in between scans. To protect sensitive detectors from the primary beam during alignment purposes (without beamstops), an attenuator box (XIA PF4 with XIA PFCU) is installed in front KB entrance slits, with four independent filters of foil thickness values raised to the power of two, and thickness/material selectable according to photon energy.

KB mirrors: The central component of the GINIX instrument is the KirkpatrickBaez (KB) focusing system providing a x-ray nano-focus for the photon energy range between $6 \mathrm{keV}-14 \mathrm{keV}$ (Kalbfleisch et al., 2011; Salditt et al., 2011), see also (Kalbfleisch, 2012) for details on design and commissioning of the system, and (Bartels, 2013) for more recent applications. The two orthogonal mirrors polished to fixed elliptical curvature are each $100 \mathrm{~mm}$ long, and accept a maximum beam size of $\simeq 0.4 \mathrm{~mm}$. The first mirror (WinlightX, France) focuses in vertical direction (VM) with focal length $f=302 \mathrm{~mm}$ and incidence angle $\theta=3.954 \mathrm{mrad}$ (mirror center) the second mirror (JTEC, Japan) focuses in horizontal direction (HM) with focal length $f=200 \mathrm{~mm}$ and incidence angle $\theta=4.05 \mathrm{mrad}$ (mirror center). Both mirrors were coated with $P d$. The figure error after coating (peak-to-valley) is $13.9 \mathrm{~nm}(\mathrm{VM})$ and $4.8 \mathrm{~nm}(\mathrm{HM})$, 
respectively. The focal field distribution has been simulated for the measured figure errors and compared to measurements of the focal intensities by waveguide scans (Salditt et al., 2011). In a current upgrade of the system, a completely new polishing and coating $(R h)$ has been performed (JTEC, Japan) achieving improved surface quality with $\leq 0.5 \mathrm{~nm}$ peak-to-valley for both mirrors, as measured by optical metrology. In addition, the $\mathrm{KB}$ mechanics is equipped with a miniaturized fiber interferometer (FPS30110, Attocube). The focal plane is at $87.6 \mathrm{~m}$ behind the source. To reduce beaminduced degradation, the KB system is operated in a ultra high vacuum vessel, with $7 \times 7 \mathrm{~mm}$ windows made of $1 \mu \mathrm{m}$ thin $S i_{3} N_{4}$ membranes (Silson, UK). The beam size impinging onto the mirrors can be controlled by different slit system: The entrance slit system of the instrument equipped hybrid metal single-crystal blades (Xenocs, France) is mounted on the optical table directly in front of the vacuum vessel, but it was found in operation that it is of advantage to define the incoming beam by the beamline's $G 2$ slit and to use the KB slit for clean-up. Alignment of the KB is typically first performed by optical inspection of the focal spot size on a scintillation foil observed with an optical microscope, followed by waveguide scans through the focus, and eventually ptychographic probe reconstruction. Depending on orbit parameters, slit settings and alignment status, focal spot sizes down to about $\simeq 200 \mathrm{~nm} \times 200 \mathrm{~nm}$ (FWHM,as measured by waveguide scans) can be achieved with a flux larger than $10^{11} \mathrm{ph} / \mathrm{s}$ (Salditt et al., 2011). Coherent illumination of the mirrors by closing the slit G2 results in focal spot sizes focus sizes in the range of $150-500 \mathrm{~nm}$ (Giewekemeyer et al., 2013; Wilke et al., 2013; Wilke et al., 2014). Note that ptychography can give spot sizes which are smaller than the waveguide scans, since values are unaffected by convolution and since the focal plane is found automatically by numerical propagation. For small slit settings, however, the focus is broadened by diffraction, as expected.

IUCr macros version 2.1.6: 2014/10/01 
(a)
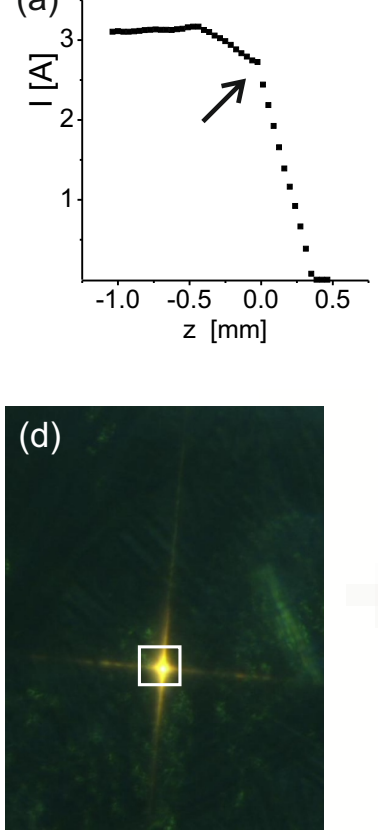

(b)

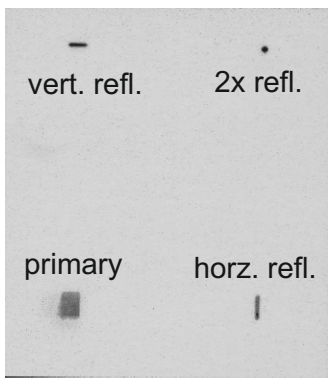

(e)

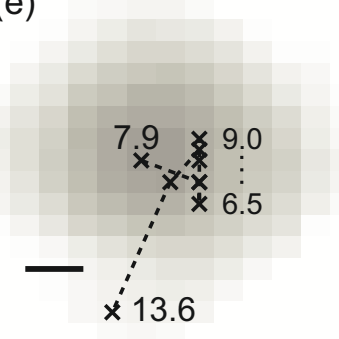

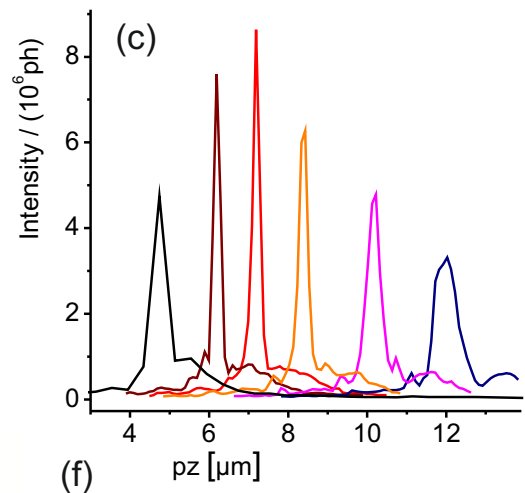

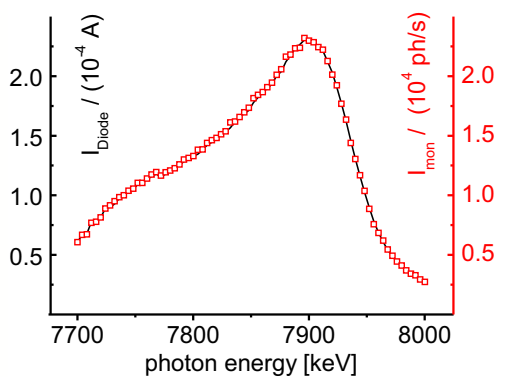

Fig. 2. Alignment of the KB mirrors. (a) Translation of the horizontal mirror (HM) along $z$ into the primary beam. The correct position for is indicated by the arrow. (b) The primary beam with the two single and the double focused reflection, as recorded with an alignment detector, positioned roughly in the focal plane. To observe the four beams, the 'heavy duty' aperture which normally served to single out only the double reflected beam, has to be moved out. A 'correct' spacing between the reflections indicates that the incidence angles on the mirror are correct. This coarse alignment is followed by the fine alignment with waveguides, illustated in (c). (c) Vertical intensity distributions in the KB focal plane scanned for precision alignment by a planar waveguide with a $35 \mathrm{~nm}$ guiding layer, for different incidence angles of the mirror (VM), covering a range of $7 \mu \mathrm{rad}$. The maximum intensity and minimum width indicates the correct incidence angle, here with a focal spot size (FWHM) of 208nm. (d-f) Data illustrating the positional stability when changing the photon energy (here from $7.9 \mathrm{keV}$ to $13.6 \mathrm{keV}$ ). The KB focal spot is converted to visible light by a scintillation sceen (d) in the focal plane which is then observed by the optical microscope. The observed trace during an energy scan of the double crystal monochromator is plotted in (e), overlaid with the intensity distribution of the $7.9 \mathrm{keV}$ beam (limited by optical resolution) as background. Scale bar: $4.8 \mu \mathrm{m}$. (f) The traces of the EH2 entrance monitor and the KB beam intensity overlay perfectly as a function of photon energy, without any further motor movements, else the scan of the double crystal monochromator, reflecting the profile of the first undulator harmonic.

Beam conditioning and waveguide optics: The first optical element behind 
the KB-system for KB-beam selection is a motorized 'heavy-duty' aperture (diameter $1 \mathrm{~mm}, 450 \mu \mathrm{m} \mathrm{W}$ ), compactly installed on the breadboard of the $\mathrm{KB}$ vessel, to block the direct and single reflected beams. Alignment of the KB is facilitated by measuring the positions of the direct beam and all three reflections by a high high resolution camera in the focal plane, see Fig.1, so that the pinhole is moved out for this purpose. In between $\mathrm{KB}$ vessel and the $\mathrm{KB}$ focal plane, three further stages for beam conditioning are installed. Two compact $x y z$-translation stages (SmarAct-XYZ SLC30s SBP), are positioned at $x=-15 \pm 16 \mathrm{~mm}$ and $x=-7 \pm 16 \mathrm{~mm}$ upstream of the KB focal plane, respectively. These stages can be used to insert/position optical elements for cleaning of the KB beam, in order to achieve sufficient suppression of tails (KB streaks) as required for high quality nano-diffraction experiments. Specially designed rectangular soft edge apertures (Takahashi et al., 2013) are available for this purpose, while pinholes fabricated by ion beam milling with diameters down to a few $\mu \mathrm{m}$ find application in ptychography (beam compactification) and holographic imaging with the KB beam. Further optical functions (wavefront masks, gratings) could also be implemented on these holders. Secondly, a hexapod system (custom designed SmarPod 110.45, Smaract) which is used to align and position the waveguides, both to scan through the KB focus for alignment or diagnostics, and for holographic imaging with the KB beam . Kinematic mounts, and tabulated position values enable fast exchange of different waveguides, facilitating waveguide alignment, which generally involves three translations and two rotations orthogonal to the optical axis. The center of rotation can be conveniently shifted to the entrance of the waveguide, if needed selectively for each individual channel of a waveguide chip. The nanopositioners are all equipped with optical encoders, enabling closed loop operation, and performance of the nanopositioners has been characterized with a Michelson interferometer (SIOS SP-S 120) (Kalbfleisch, 2012). Recent re-inspection of the vibrations of the sample

IUCr macros version 2.1.6: 2014/10/01 
tower showed vibration amplitudes on the order of 50nm (rms), making it highly likely that the current resolution in waveguide holography is limited by vibrations. An upgrade directed to vibration isolation by means of a monolithic high-resolution stage for waveguide optics (hexapod) and sample is therefore in planning.

Optical microscopes: The alignment of optical components (waveguides, cleanup pinholes) and of the samples is facilitated by two optical on-axis microscopes (Kalbfleisch, 2012), which are directed parallel (pre-focus) and anti-parallel (postfocus) to the beam. Both microscopes are compatible with in-situ x-ray recordings, but are also fully motorized $(x y z)$ to be moved out of the beam. The first (prefocus) microscope (Optique Peter) is equipped with a long-distance $(50 \mathrm{~mm})$ objective, motorized zoom, and uses a tilted mirror with a drilled hole (diameter $1.5 \mathrm{~mm}$ ) reflect the optical light out of the x-ray optical axis. The second microscope (post-focus) is the on-axis video microscope (OAV, Bruker ASC), equipped with motorized zoom, and a drilled objective (diameter $1.2 \mathrm{~mm}$ ) of $32 \mathrm{~mm}$ working distance, and hence compatible with observations of cryogenically cooled samples or larger sample chambers.

Instrument control: The GINIX control system is implemented based on the SPEC software by CSS (Certified Scientific Software, 2015). From the SPEC command line, all relevant motors can be controlled and more than two dozen counters can be accessed; also, analysis software is connected via network links for live feedback. In total, more than 130 degrees of freedom ranging from stepper motors, piezos and macro motors to beamline motors accessed via Tango can be controlled and scanned in parallel. About 30 counters from simple diodes over MCA channels to pixel detector statistics (total/ROI intensity, centre of mass, peak width) can be queried and used for alignment. Pixel detector images (Pilatus, Maxipix etc.) are shown on a separate screen as soon as they are available, in linear and logarithmic colour scales; STXM measurements are instantly analysed. Also, flat field correction and live holographic

IUCr macros version 2.1.6: 2014/10/01 
reconstruction are available. Pixel detectors can also be used during line scans, and commands to move motors to maximum, minimum and centre-of-mass positions are implemented. Detector images as well as images from two optical inline microscope can be printed for the paper logbook with a single command, also from a measurement macro. The optical microscopes support aligning the sample with respect to the x-ray beam, with overlaying markers for the focus position. Images can be saved during a scan, in parallel to the x-ray acquisition. Apart from purely software-controlled scans with motor settling, also a continuous (fly) scan of the piezo stage with hardwaretriggered detector exposure is available. This allows for $2 \mathrm{D}$ raster scanning with typically $(100-200)^{2}$ positions and detector images with the Pilatus 300k within five to twenty minutes. From this data, high-resolution STXM images with user-defined contrast (e.g. differential phase contrast, Bragg peak position and size, streak finder) can be calculated and correlated with optical microscopy.

\section{References}

Balewski, K., Brefeld, W., Decking, W., Franz, H., Röhlsberger, H. \& Weckert, E. (2004). PETRA III: A Low Emittance Synchrotron Radiation Source. Tech. rep. Deutsches Elektronen-Synchrotron DESY.

Bartels, M. (2013). Cone-beam x-ray phase contrast tomography of biological samples Optimization of contrast, resolution and field of view. Ph.D. thesis, Universität Göttingen.

Bartels, M., Priebe, M., Wilke, R., Krüger, S., Giewekemeyer, K., Kalbfleisch, S., Olendrowitz, C, C., Sprung, M. \& Salditt, T. (2012). Opt. Nanoscopy, 1(1), 10.

Certified Scientific Software, (2015). http://certif.com.

Giewekemeyer, K., Philipp, H. T., Wilke, R. N., Aquila, A., Osterhoff, M., Tate, M. W., Shanks, K. S., Zozulya, A. V., Salditt, T., Gruner, S. M. \& Mancuso, A. P. (2014). J. Synchrotron Rad. 21(5), 1167-1174.

Giewekemeyer, K., Wilke, R. N., Osterhoff, M., Bartels, M., Kalbfleisch, S. \& Salditt, T. (2013). J. Synchrotron Rad. 20(3), 490-497.

Kalbfleisch, S. (2012). A Dedicated Endstation for Waveguide-based X-Ray Imaging. Ph.D. thesis, Universität Göttingen.

Kalbfleisch, S., Neubauer, H., Krüger, S. P., Bartels, M., Osterhoff, M., Mai, D. D., Giewekemeyer, K., Hartmann, B., Sprung, M. \& Salditt, T. (2011). AIPConf.Proc. 1365(1), 96-99.

Leupold, O. \& Sprung, M. (2008). Coherence Beamline at PETRA III - Beamline Design Report. Tech. rep. Deutsches Elektronen-Synchrotron DESY.

Olendrowitz, C., Bartels, M., Krenkel, M., Beerlink, A., Mokso, R., Sprung, M. \& Salditt, T. (2012). Phys. Med. Biol. 57(16), 5309.

Pennicard, D., Lange, S., Smoljanin, S., Hirsemann, H. \& Graafsma, H. (2012). Journal of Instrumentation, 7(11), C11009.

IUCr macros version 2.1.6: 2014/10/01 
Rossi, L., Fischer, P., Rohe, T. \& Wermes, N. (2006). Pixel Detectors: From Fundamentals to Applications. Springer.

Salditt, T., Kalbfleisch, S., Osterhoff, M., Krüger, S. P., Bartels, M., Giewekemeyer, K., Neubauer, H. \& Sprung, M. (2011). Opt. Express, 19(10), 9656-9675.

Takahashi, Y., Suzuki, A., Furutaku, S., Yamauchi, K., Kohmura, Y. \& Ishikawa, T. (2013). Appl. Phys. Lett. 102(9), 094102-4.

Wilke, R. N., Hoppert, M., Krenkel, M., Bartels, M. \& Salditt, T. (2014). J. Appl. Crystallogr. 48, 464-476.

Wilke, R. N., Vassholz, M. \& Salditt, T. (2013). Acta Crystallogr.A, 69(5), 490-497.

IUCr macros version 2.1.6: 2014/10/01 